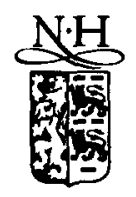

ELSEVIER

Applied Surface Science 92 (1996) 532-536

applied

surface science

\title{
Noise characteristics of an infrared hot-electron transistor
}

\author{
C.H. Kuan * \\ Department of Electrical Engineering, National Taiwan University, Taipei, Taiwan, ROC
}

Received 12 December 1994; accepted for publication 2 March 1995

\begin{abstract}
We report on the noise characteristics of dark current in an infrared hot-electron transistor at $77 \mathrm{~K}$. The measured emitter noise power can be described as the sum of generation-recombination noise and thermal noise. The measured collector noise in our device is shot noise. This result is explained with the binomial statistics for the number of injected electrons to pass through the base and the energy filter. Since the probability for the electrons contributing to the collector current is much less than 1 in our noise measurement range, the binomial statistics can be approximated as Poisson statistics and the noise of the collector current behaves like shot noise.
\end{abstract}

A novel quantum well infrared photodetector (QWIP) based on intersubband transitions was first proposed by West and Eglash [1] and has drawn special attention because of its physical origin and mature growth Technology. The main concern about a QWIP, used as a pixel of a focal plane array, is the dark current at high temperature. In particular, at 77 $\mathrm{K}$ the dominant thermal current is so large that it saturates the read-out circuit [2]. A promising device which has smaller dark current and hence can be operated at $77 \mathrm{~K}$ with background limited performance is the infrared hot-electron transistor (IHET) $[3,4]$. In this paper, we report about the noise characteristics of the dark current in an IHET at $77 \mathrm{~K}$ and establish a model to explain the noise performance.

The IHET we measure is a three-terminal device as shown in Fig. 1. Between the emitter (E) and base

\footnotetext{
* Corresponding author. Tel: +886-2-3635251; fax: + 886-23638247 .
}

(B), there is a QWIP which is followed by an energy filter and a collector contact. At $77 \mathrm{~K}$, the dominant dark current in a QWIP under a fixed bias $V_{\mathrm{BE}}$ is due to thermally assisted tunneling [5]. Those thermally excited electrons first tunnel through the adjacent barrier and then travel above the succeeding wells and barriers. During the process, they release their energy by scattering and are finally either trapped in another well or collected by the base contact. The noise due to those eletrons can be described by generation-recombination noise ( $G-R$ noise), $4 e g I_{\mathrm{E}}$, where $e$ is the electron charge, $g$ is the current gain of the photocurrent, and $I_{\mathrm{E}}$ is the emitter current [6-8]. Strictly and theoretically speaking, the $G-R$ noise power of the photocurrent $I_{\mathrm{p}}$ is $4 e g I_{\mathrm{p}}$ while that of the dark current should be $4 e g I_{\mathrm{E}} F$ where $F$ is a correction factor [9]. If the capture probability of the electron in the quantum well is much less than $1, F$ can be approximated as 1. In our sample, the excited state in the quantum well is above the barriers and the capture probability 


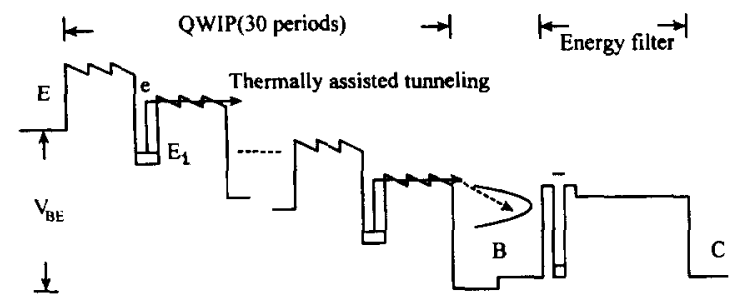

Fig. 1. Band diagram of the infrared hot-electron transistor under a fixed bias $V_{\mathrm{BE}} . \mathrm{E}, \mathrm{B}$ and $\mathrm{C}$ denote the emitter, the base and the collector respectively. $E_{1}$ is the ground state energy in the quantum wells.

is expected to be small. The experimental results of our sample agree with this expectation.

Another noise source in the QWIP is the thermal noise which is determined by the differential conductance [7] and electron temperature. The relaxation of the electron energy by scattering may render the temperature of electrons to be the same as the crystal. We find that the thermal noise in our device can be estimated with the crystal temperature.

The electrons injected from the QWIP traverse the base and tunnel through the energy filter. This process causes the number of electrons collected by the collector to follow a statistical distribution. Therefore, in addition to the emitter noise, the noise performance of the collector is affected by the energy filter. In our device, we find that the probability of the electrons contributing to the collector current is much less than 1 in our noise measurement range. The scattering process within the base and the tunneling process through the energy filter dominate the emitter noise and hence the collector noise behaves like shot noise.

Our IHET is made of GaAs/AlGaAs. The barriers of the QWIP between the emitter and base have three steps which are 161,167 , and $173 \AA$ thick respectively. The corresponding aluminum concentrations are $0.28,0.305$, and 0.33 . The GaAs well of the QWIP is $50 \AA$ thick and doped with $n=1.2 \times$ $10^{18} \mathrm{~cm}^{-3}$ of $\mathrm{Si}$. The period number of the QWIP is 30. The emitter contact is composed of $6000 \AA \mathrm{GaAs}$ doped with $n=1.2 \times 10^{18} \mathrm{~cm}^{-3}$. The base contact consists of $300 \AA \mathrm{In}_{0.1} \mathrm{Ga}_{0.9}$ As and $200 \AA$ GaAs both doped with $n=1.0 \times 10^{18} \mathrm{~cm}^{-3}$. Between the base and the collector (C), there is an double-barrier structure, which form a band pass filter with a pass band centered at $260 \mathrm{meV}$ and a bandwidth of 25 meV to cut down the dark current. The composition of the energy filter is $40 \AA \mathrm{Al}_{0.3} \mathrm{Ga}_{0.7} \mathrm{As}, 50 \AA \mathrm{GaAs}$ and $40 \AA \mathrm{Al}_{0.3} \mathrm{Ga}_{0.7} \mathrm{As}$ followed by $2000 \AA$ $\mathrm{Al}_{0.25} \mathrm{Ga}_{0.75}$ As. The collector contact is made of $11000 \AA$ GaAs doped with $n=1.0 \times 10^{18} \mathrm{~cm}^{-3}$.

During the characterization of the IHET at $77 \mathrm{~K}$, the QWIP is under a fixed bias $V_{\mathrm{BE}}$ between the base and emitter while the base and the collector are always kept at the same ground potential as shown in Fig. 1. The emitter dark current density $J_{\mathrm{E}}$ and the

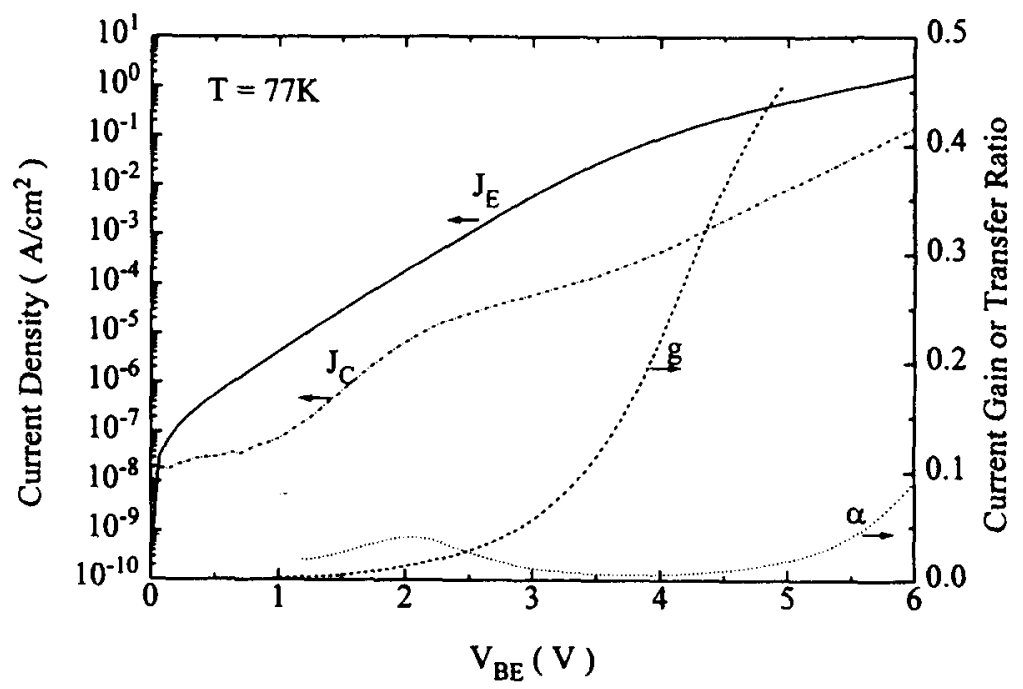

Fig. 2. Dark current densities $\left(J_{\mathrm{E}}\right.$ and $J_{\mathrm{C}}$ ), the emitter current gain $(\mathrm{g})$, and the transfer ratio $(\alpha)$ as functions of the applied bias $V_{\mathrm{BE}}$. 


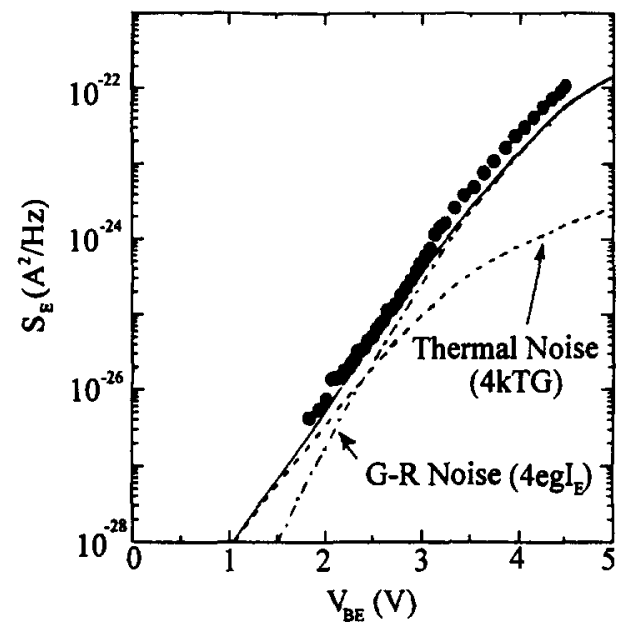

Fig. 3. Emitter current noise power $\left(S_{\mathrm{E}}\right)$ versus $V_{\mathrm{BE}}$ (solid circles). The dash-dotted curve represents the generation-recombination noise power and the dashed curve stands for the thermal noise power. The solid curve describes the sum of the two noise powers.

collector dark current density $J_{\mathrm{C}}$ are shown in Fig. 2 . In the noise measurement, the noise signal in the emitter current $\left(I_{\mathrm{E}}\right)$ is amplified by a voltage amplifier and that in the collector current $\left(I_{C}\right)$ is amplified by a current amplifier. The noise sources of the two amplifiers and the background noise in the circuit are well calibrated. The differential resistances of the emitter and collector are also measured. The frequency range of our measurements is from $0 \mathrm{~Hz}$ to $100 \mathrm{kHz}$. The current noise powers $\left(S_{\mathrm{E}}\right.$ and $\left.S_{\mathrm{C}}\right)$ at the emitter and collector are calculated from the measurement results and the equivalent circuits of the amplifiers and the IHET [10]. The solid circles in Figs. 3 and 4 describe the experimental results of $S_{\mathrm{E}}$ and $S_{\mathrm{C}}$ as functions of $V_{\mathrm{BE}}$, respectively. As expected, the dark current in the emitter and collector increases with increasing bias $V_{\mathrm{BE}}$, as shown in Fig. 2 , and both current noise powers also increase with increasing bias.

In the following, we first discuss the noise performance of the collector and then that of the emitter. As shown in Fig. 4, the solid curve represents the shot noise power $2 e I_{C}$ of the collector current as a function of $V_{\mathrm{BE}}$. It agrees with the experimental results very well. The physical origin of the shot noise can be explained with the binomial statistics. As shown in Fig. 1, the electrons are injected from the QWIP and scattered by impurities and phonons in the base. Upon the impact of the energy filter, the electrons have to tunnel through the barriers.

Let us assume that within the measurement time $\tau$ (i.e., the sampling time of the noise signal), there are $N_{\mathrm{E}}$ electrons injected from the QWIP. The probability that the electrons can pass through the base and energy filter is $p$. Then the collector current can be written as

$I_{\mathrm{C}}=\frac{e \overline{N_{\mathrm{C}}}}{\tau}=\frac{e \overline{N_{\mathrm{E}}} p}{\tau}$,

where $N_{\mathrm{C}}$ is the number of electrons collected at the collector, and 'overstrike' denotes the average value. According to binomial statistics, the variance $\overline{\Delta N_{\mathrm{C}}^{2}}$ is

$\overline{\Delta N_{\mathrm{C}}^{2}}=\overline{\Delta N_{\mathrm{E}}^{2}} p^{2}+\overline{N_{\mathrm{E}}} p(1-p)$.

If $N_{\mathrm{E}}$ follows the Poisson statistics, the variance is $\overline{\Delta N_{\mathrm{E}}^{2}}=\overline{N_{\mathrm{E}}}$. If not, we expect that $\overline{\Delta N_{\mathrm{E}}^{2}}$ is less than or equal to $\overline{N_{\mathrm{E}}}$ because the measured emitter noise is less than shot noise ( $g<0.5$ as shown in Fig. 2). Under the condition $p \ll 1$, we have $\overline{\Delta N_{C}^{2}} \approx \overline{N_{E}} p$ $=\overline{N_{\mathrm{C}}}$ and the variance of the collector current is

$\Delta I_{\mathrm{C}}^{2}=\frac{e^{2}}{\tau^{2}} \overline{\Delta N_{\mathrm{C}}^{2}}=\frac{e}{\tau} I_{\mathrm{C}}$.

According to the sampling theorem [12], the measured maximum frequency $B$ with the measurement time $\tau$ is $1 / 2 \tau$. Hence, the current noise power $S_{\mathrm{C}}$ is

$S_{\mathrm{C}}=\frac{\Delta I_{\mathrm{C}}^{2}}{B}=2 e I_{\mathrm{C}}$

which represents shot noise.

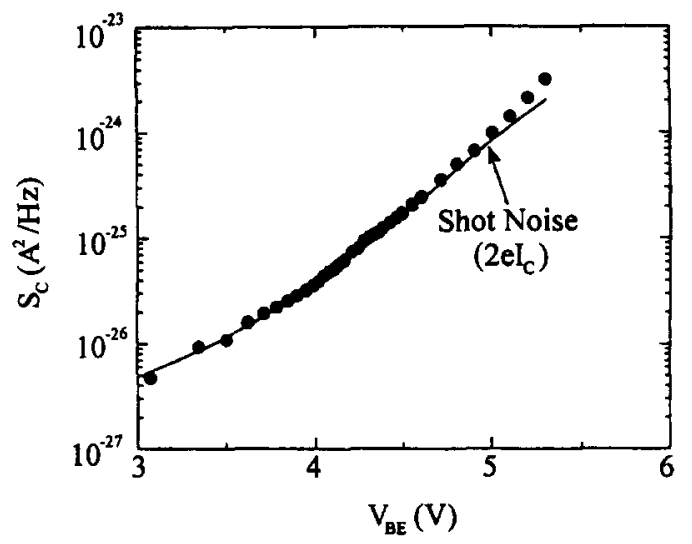

Fig. 4. Collector current noise power $\left(S_{C}\right)$ versus $V_{B E}$ (solid circles). The solid curve represents the shot noise power. 
The assumption $p \ll 1$ is confirmed by the transfer ratio $\alpha=I_{\mathrm{C}} / I_{\mathrm{E}}$ since $I_{\mathrm{E}}=\mathrm{e} \overline{N_{\mathrm{E}}} / \tau$ and $\alpha=p$. The experimental result of $\alpha$ is shown in Fig. 2 . Within the bias range of the noise measurement, $V_{\mathrm{BE}}=3-5 \mathrm{~V}$, the value of $\alpha$ is $0.01-0.02$. This indicates that the probability $p$ for electrons contributing to the collector current is much less than 1 . Therefore, the experimental results are consistent with the model. In other words, if $p \ll 1$, the noise current of the emitter cannot flow through the energy filter. The collector noise dominated by the scattering and tunneling processes is shot noise. In addition, we should notice that when $V_{\mathrm{BE}}>5 \mathrm{~V}$, the measured noise power becomes to be larger than that of shot noise. This is consistent with the increasing trend of the transfer ratio in Fig. 2. The noise in the emitter becomes influential on the collector current when $V_{\mathrm{BE}}>5 \mathrm{~V}$.

According to a law of stochastic process [12], the binomial statistics can be approximated as Poisson statistics if $p$ is much less than 1. The noise for the Poisson statistics is shot noise [7]. Additionally it follows from the theoretical analysis of the transmission probability through a barrier [13], assuming the transmission probability is very small, that the noise performance for the tunneling is shot noise. Both of these mathematical and physical arguments are consistent with our results.

For the emitter part, in order to find out the relationship between $I_{\mathrm{E}}$ and $S_{\mathrm{E}}$, we also measure the responsivity $R_{\mathrm{p}}$ of the photocurrent in the emitter at $77 \mathrm{~K}$ and the quantum efficiency $\eta$, which is $14 \%$ for our device. From these two results and as shown in Fig. 2, we derive the current gain $g$ :

$g=\frac{R_{\mathrm{p}} h \nu}{\eta e}$,

where $\nu$ is the frequency of the input photon. The G-R noise power $4 e g I_{\mathrm{E}}$ of the emitter current is represented by the dash-dotted curve in Fig. 3. It agrees with $S_{\mathrm{E}}$ at $V_{\mathrm{BE}} \geqslant 3 \mathrm{~V}$. At low biases, the G-R noise power deviates from the solid circles. From the measured differential emitter conductance $G$, we also estimate the thermal noise power $4 k T G$ with $T=77 \mathrm{~K}$. The result is shown with the dashed curve in the same figure. The solid circles match well with the solid curve which is the sum of thermal and $\mathrm{G}-\mathrm{R}$ noise powers.
At $77 \mathrm{~K}$, the dominant current in the QWIP is due to the thermally assisted tunneling electrons. Those electrons can become hot due to the externally applied field. However, it is possible for them to release their energy due to the scattering at the three-step barriers and at optical phonons. If this happens, the temperature of the electrons can be the same as crystal atoms. We compare the current density of the three-step QWIP with the conventional square-barrier QWIP under the same electrical field on the barriers and find the current density of the three-step QWIP is at least two orders of magnitude smaller. From the calculation for the tunneling probability, we find that the longer tunneling length in the three-step barriers causes the electron density above the barriers to become of one order less. Since the current density is the product of the electron density and the drift velocity, we may attribute the other order of magnitude less to the scattering of the three-step barriers when the electrons are travelling above the barriers. This strong scattering causes the electron to release their energy and hence we can use the temperature of crystals to estimate the thermal noise power of the emitter. The agreement of the data confirms our judgement.

Besides thermal noise, most of the electrons generated by thermally assisted tunneling are finally trapped in another quantum well. The noise due to this mechanism can be described by G-R noise, since those electrons behave similar to photo-generated electrons. Therefore, we expect that the current gains of both kinds of electrons are the same. The $\mathrm{G}-\mathrm{R}$ noise can hence be written as $4 e g I_{\mathrm{E}}$ and the experimental results agree with $G-R$ noise at high bias voltages. As shown in Fig. 2, the current gain is very small when $V_{\mathrm{BE}} \leqslant 2.5 \mathrm{~V}$ and increases with increasing bias. Since the current gain increases very rapidly for $V_{\mathrm{BE}} \geqslant 2.5 \mathrm{~V}, \mathrm{G}-\mathrm{R}$ noise becomes to dominate thermal noise at $V_{\mathrm{BE}}=2.5 \mathrm{~V}$.

In summary, we have measured the noise of an IHET and found that the noise power at the collector can be explained as shot noise if the probability for the injected electrons from the QWIP contributing to the collector current is very small. We used the binomial statistics as a model to describe the noise behavior of the electrons. As the probability is much less than 1, binomial statistics can be described by the Poisson statistics, and hence the noise is associ- 
ated with shot noise. At the emitter, the noise power can be written as the sum of thermal noise and $G-R$ noise. G-R noise is dominant over thermal noise at high biases because of the high current gain.

We would like to thank Professor D.C. Tsui at Princeton University and Dr. K.K. Choi at US Army Laboratory for helpful discussion, and Dr. Yuan P. $\mathrm{Li}$ at AT \& T Bell Laboratory for the assistance in the experiment.

\section{References}

[1] L.C. West and S.J. Eglash, Appl. Phys. Lett. 46 (1985) 1156.

[2] C.G. Bethea and B.F. Levine, Proc. SPIE 1735 (1992) 198.

[3] K.K. Choi, M. Dutta, P.G. Newman and M.-L. Saunders, Appl. Phys. Lett. 57 (1990) 1348.
[4] K.K. Choi, L. Fotiadis, M. Taysing-Lara and W. Chang, Appl. Phys. Lett. 60 (1992) 592.

[5] C.H. Kuan, D.C. Tsui, K.K. Choi, P.G. Newman and W.H. Chang, Appl. Phys. Lett. 63 (1993) 2091.

[6] A. Rose, Concepts in Photoconductivity and Allied Problems (Interscience, New York, 1963).

[7] A. van der Ziel, Noise in Solid State Devices and Circuits (Wiley, New York, 1986).

[8] G. Hasnain, B.F. Levine, S. Gunapala and N. Chand, Appl. Phys. Lett. 57 (1990) 608.

[9] K.K. Choi, Appl. Phys. Lett. 65 (1994) 1266.

[10] Y.P. Li, A. Zaslavsky, D.C. Tsui, M. Santos and M. Shayegan, Phys. Rev. B 41 (1990) 8388; in: Resonant Tunneling in Semiconductors, Ed. L.L. Chang et al. (Plenum, New York, 1991).

[11] A.V. Oppenheim and A.S. Willsky, Signals and Systems (Prentice-Hall, Englewood Cliffs, NJ, 1983) p. 519.

[12] C.V. Heer, Statistical Mechanics, Kinetic Theory, and Stochastic Processes (Academic Press, New York, 1972) pp. 84-86.

[13] B. Yurke and G.P. Kochanski, Phys. Rev. B 41 (1990) 8184. 